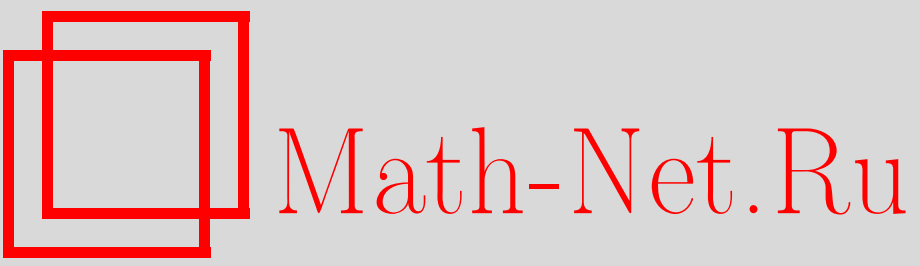

Р. М. Кашаев, Уравнение пятиугольника и группы классов отображений поверхностей с отмеченными точками, ТМФ, 2000, том 123, номер 2, 198-204

DOI: https://doi.org/10.4213/tmf598

Использование Общероссийского математического портала Math-Net.Ru подразумевает, что вы прочитали и согласны с пользовательским соглашением

http://www . mathnet.ru/rus/agreement

Параметры загрузки:

IP : 54.196 .121 .252

26 апреля 2023 г., 15:31:49 
ТЕОРЕТИЧЕСКАЯ

И МАТЕМАТИЧЕСКАЯ

ФИЗИКА

Том 123, № 2

май, 2000

(C) 2000 г.

P. M. Кашаев*

\section{УРАВНЕНИЕ ПЯТИУГОЛЬНИКА И ГРУППЫ КЛАССОВ ОТОБРАЖЕНИЙ ПОВЕРХНОСТЕЙ С ОТМЕЧЕННЫМИ ТОЧКАМИ}

В квантовой теории Тейхмюллера, основанной на координатах Пеннера, реализуется проективное представление групп классов отображений поверхностей с отмеченными точками. Можно заметить, что алгебраически это представление основывается на уравнении пятиугольника и двух дополнительных соотношениях. Два примера решений этих уравнений связаны с матричными (или операторными) обобщениями дилогарифмма Роджера. Соответствуюшие центральные заряды рациональны. Возможно, что эта система уравнений допускает много различных решений.

\section{1. ВВЕДЕНИЕ}

Пусть $V$ - векторное пространство, а обратимые элементы $\mathbf{T} \in \operatorname{End}(V \otimes V), \mathbf{R} \in$ $\operatorname{End}(V)$ удовлетворяют следуюшей системе уравнений:

$$
\begin{gathered}
\mathbf{T}_{12} \mathbf{T}_{13} \mathbf{T}_{23}=\mathbf{T}_{23} \mathbf{T}_{12}, \\
{[\mathbf{R} \otimes 1 \mathbf{T} 1 \otimes \mathbf{R}, \mathbf{P}]=0,} \\
\mathbf{T R} \otimes 1 \mathbf{P T}=\zeta \mathbf{R} \otimes \mathbf{R}, \\
\mathbf{R}^{3}=1,
\end{gathered}
$$

где $\zeta$ - комплексное число на единичной окружности, $\mathbf{P} \in \operatorname{End}(V \otimes V)$ - оператор перестановки, определяемый соотношением $\mathbf{P} x \otimes y=y \otimes x$, а $\mathbf{T}_{13}$ является ограничением элемента $\mathbf{T}$ на первый и третий сомножители тензорного произведения $V \otimes V \otimes V$ и т.д. Было показано $[1,2]$, что в квантовой теории пространств Тейхмюллера поверхностей с отмеченными точками эта система уравнений позволяет построить проективное представление группы классов отображений поверхностей с отмеченными точками. В этом случае проективный множитель является целой степенью числа $\zeta$.

В квантовой конформной теории поля группы классов отображений имеют проективное представление в пространстве конформных блоков, при этом проективный множитель связан с центральным зарядом алгебры Вирасоро. В этом смысле центральньй

* Математический институт им. В. А. Стеклова РАН, Санкт-Петербургское отделение, СанктПетербург, Россия; Хельсинкский институт физики, Хельсинкский университет, Хельсинки, Финляндия. E-mail: Rinat.Kashaev@helsinki.fi 
заряд алгебры Вирасоро (гипотетической) конформной теории поля, в которой соответствуюшее представление группы классов отображений задается системой уравнений (1)-(4), связан с числом $\zeta$ формулой

$$
\exp (i \pi c)=\zeta^{-72}
$$

Цель данной работы состоит в описании некоторых решений системы (1)-(4).

\section{2. ПОВЕРХНОСТЬ РОДА ТРИ С ОДНОЙ ОТМЕЧЕННОЙ ТОЧКОЙ}

В этом разделе показано, как соотношения (1)-(4) приводят к проективному представлению группы классов отображений поверхности рода три с одной отмеченной точкой. Если род поверхности меньше трех, проективный множитель соответствуюшей группы классов отображений можно устранить переопределением генераторов. В случае рода три и выше определяюшие соотношения для генераторов (которые являются твистами Дена вдоль неразделяюших кривых) включают сплетаюшие соотношения, фиксируюшие относительную нормировку генераторов; “фонарное” соотношение, фиксируюшее оставшуюся свободу в нормировке генераторов, и "цепное" соотношение, выявляюшее проективный множитель. Случай поверхности рода три с одной отмеченной точкой был исследован в работе [2].

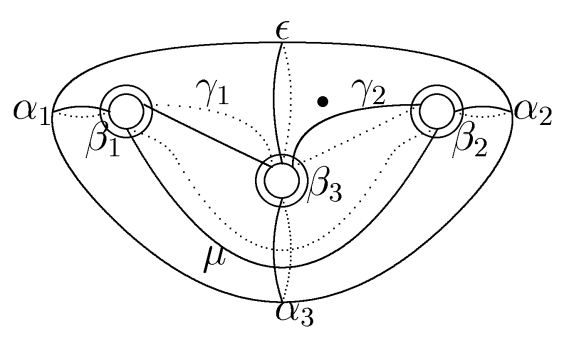

Рассмотрим кривые на поверхности, показанной на рисунке. Твисты Дена вдоль указанных кривых порождают группу классов отображений этой поверхности и задаются в тензорном произведении $V^{\otimes 10}$ (множители нумеруются числами $\left.0,1, \ldots, 9\right)$ следующими формулами:

$$
\begin{aligned}
& \mathbf{F}_{\alpha_{i}}=\zeta^{-6} \operatorname{Ad}\left(\mathbf{T}_{3 i-1,3 i-2}\right) \mathbf{T}_{3 i, \widehat{3 i-2}}^{-1}, \\
& \mathbf{F}_{\beta_{i}}=\zeta^{-6} \operatorname{Ad}\left(\mathbf{T}_{3 i, \widehat{3 i-2}}\right) \mathbf{T}_{3 i-1,3 i}^{-1}, \quad i=1,2,3 \text {, } \\
& \mathbf{F}_{\gamma_{1}}=\zeta^{-6} \operatorname{Ad}\left(\mathbf{T}_{0 \check{7}} \mathbf{T}_{87} \mathbf{T}_{10} \mathbf{T}_{1 \check{g}} \mathbf{T}_{21} \mathbf{T}_{\check{3} 1} \mathbf{T}_{27} \mathbf{T}_{30}\right) \mathbf{T}_{\check{0} \hat{7}}^{-1}, \\
& \mathbf{F}_{\gamma_{2}}=\zeta^{-6} \operatorname{Ad}\left(\mathbf{T}_{87} \mathbf{T}_{80} \mathbf{T}_{84} \mathbf{T}_{\hat{8} 9} \mathbf{T}_{69} \mathbf{T}_{\check{9} 7} \mathbf{T}_{54} \mathbf{T}_{\check{7} 8}\right) \mathbf{T}_{\check{8} \hat{4}}^{-1}, \\
& \mathbf{F}_{\mu}=\zeta^{-6} \operatorname{Ad}\left(\mathbf{T}_{0 \check{7}} \mathbf{T}_{87} \mathbf{T}_{10} \mathbf{T}_{1 \grave{9}} \mathbf{T}_{\hat{8} 9} \mathbf{T}_{21} \mathbf{T}_{49} \mathbf{T}_{69} \mathbf{T}_{54} \mathbf{T}_{5 \check{8}} \mathbf{T}_{08} \mathbf{T}_{30} \mathbf{T}_{34} \mathbf{T}_{\hat{4} 9} \mathbf{T}_{\hat{1} 0}\right) \mathbf{T}_{\check{1} \hat{\rho}}^{-1}, \\
& \mathbf{F}_{\alpha_{3} \mu}=\zeta^{-6} \operatorname{Ad}\left(\mathbf{T}_{0 \check{7}} \mathbf{T}_{87} \mathbf{T}_{10} \mathbf{T}_{\hat{8} 9} \mathbf{T}_{18} \mathbf{T}_{21} \mathbf{T}_{49} \mathbf{T}_{69} \mathbf{T}_{30} \mathbf{T}_{97} \mathbf{T}_{4 \check{2}} \mathbf{T}_{54} \mathbf{T}_{\hat{4} 7} \mathbf{T}_{\hat{1} 0} \mathbf{T}_{50}\right) \mathbf{T}_{\check{0} \hat{7}}^{-1}, \\
& \mathbf{F}_{\epsilon}=\zeta^{-6} \operatorname{Ad}\left(\mathbf{T}_{0 \check{7}} \mathbf{T}_{87} \mathbf{T}_{10} \mathbf{T}_{30} \mathbf{T}_{\check{2} 0} \mathbf{T}_{\hat{3} 0} \mathbf{T}_{21} \mathbf{T}_{\hat{3} 2} \mathbf{T}_{93} \mathbf{T}_{\check{3} 1} \mathbf{T}_{\check{10}}\right) \mathbf{T}_{\check{0} \hat{7}}^{-1},
\end{aligned}
$$

где

$$
\operatorname{Ad}(a) b \equiv a b a^{-1}, \quad a_{\hat{k}} \equiv \operatorname{Ad}\left(\mathbf{R}_{k}\right) a_{k}, \quad a_{\check{k}} \equiv \operatorname{Ad}\left(\mathbf{R}_{k}^{-1}\right) a_{k}
$$


Относительная нормировка этих операторов фиксируется соотношениями вида

$$
\mathbf{F}_{\alpha_{1}} \mathbf{F}_{\beta_{1}} \mathbf{F}_{\alpha_{1}}=\mathbf{F}_{\beta_{1}} \mathbf{F}_{\alpha_{1}} \mathbf{F}_{\beta_{1}}, \ldots
$$

в то время как общая нормировка фиксируется фонарным соотношением

$$
\mathbf{F}_{\alpha_{3}} \mathbf{F}_{\mu} \mathbf{F}_{\alpha_{3} \mu}=\mathbf{F}_{\alpha_{1}} \mathbf{F}_{\alpha_{2}} \mathbf{F}_{\gamma_{1}} \mathbf{F}_{\gamma_{2}}
$$

Тем самым нормировка полностью определяется. Проверка цепного соотношения позволяет восстановить проективный множитель

$$
\left(\mathbf{F}_{\alpha_{1}} \mathbf{F}_{\beta_{1}} \mathbf{F}_{\gamma_{1}}\right)^{4}=\xi_{\mathbf{F}} \mathbf{F}_{\epsilon} \mathbf{F}_{\alpha_{3}}, \quad \xi_{\mathbf{F}}=\zeta^{-72}
$$

\section{3. ПРИМЕРЫ}

ПримеР 1. Рассмотрим некомпактный квантовый дилогарифм [3]

$$
\psi(x) \equiv \exp \left(-\frac{1}{4} \int_{-\infty}^{+\infty} \frac{\exp (-2 i x z) d z}{\operatorname{sh}(z \lambda) \operatorname{sh}\left(\frac{z}{\lambda}\right) z}\right),
$$

сингулярность при $z=0$ смешена ниже контура интегрирования, а $\lambda$ - действительный параметр. Эта функция является решением пары функциональных уравнений

$$
\psi\left(x+\frac{i \lambda^{ \pm 1}}{2}\right)=\left(1+\exp \left(2 \pi x \lambda^{ \pm 1}\right)\right) \psi\left(x-\frac{i \lambda^{ \pm 1}}{2}\right)
$$

и удовлетворяет "соотношению инверсии"

$$
\psi(x) \psi(-x)=\exp \left(-i \pi x^{2}-i \pi \frac{\lambda^{2}+\lambda^{-2}}{12}\right) .
$$

Пусть векторное пространство $V=L^{2}(\mathbb{R})$ является пространством представления алгебры Гейзенберга самосопряженных операторов с соотношением коммутации

$$
[\mathbf{p}, \mathbf{q}]=\frac{1}{2 \pi i}
$$

Тогда функция (7) удовлетворяет также операторному пятичленному тождеству

$$
\psi(\mathbf{q}) \psi(\mathbf{p})=\psi(\mathbf{p}) \psi(\mathbf{p}+\mathbf{q}) \psi(\mathbf{q})
$$

Используя эти соотношения, легко проверить, что решением системы уравнений (1)-(4) являются унитарные операторы

$$
\begin{aligned}
& \mathbf{T} \equiv \exp (2 \pi i \mathbf{p} \otimes \mathbf{q}) \psi(\mathbf{q} \otimes 1+1 \otimes(\mathbf{p}-\mathbf{q})) \\
& \mathbf{R}=\exp \left(2 \pi i \mathbf{q}^{2}-\frac{\pi i}{3}\right) \exp \left(\pi i \mathbf{p}^{2}\right) \exp \left(\pi i \mathbf{q}^{2}\right)
\end{aligned}
$$

при этом

$$
\zeta=\exp \left(-\pi i \frac{\left(\lambda+\lambda^{-1}\right)^{2}}{12}\right)
$$


Заметим, что оператор $\mathbf{R}$ удовлетворяет уравнениям

$$
\mathbf{R q R}^{-1}=\mathbf{p}-\mathbf{q}, \quad \mathbf{R p R}^{-1}=-\mathbf{q} .
$$

Этот пример взят из квантовой теории Тейхмюллера, он относится также к квантовой теории Лиувилля. В частности, центральный заряд теории Лиувилля $c_{\mathrm{L}}=1+6(\lambda+$ $\left.\lambda^{-1}\right)^{2}$ связан с $\zeta$ формулой $\exp \left(i \pi c_{\mathrm{L}}\right)=-\zeta^{-72}$ (подробности см. в [2]).

Специальный класс примеров можно построить с помощью матричных обобщений дилогарифма Роджера. Последние определяются следуюшим образом.

Пусть $I=(0,1)$ - открытый единичный интервал на действительной прямой, а $U$ - векторное пространство. Отображение $T: I \rightarrow \operatorname{End}(U \otimes U)$ называется матричным (операторным) дилогарифмом, если оно удовлетворяет следующему уравнению пятиугольника:

$$
T_{23}(x) T_{12}(y)=T_{12}\left(\frac{y-x y}{1-x y}\right) T_{13}(x y) T_{23}\left(\frac{x-x y}{1-x y}\right) .
$$

Мы рассмотрим решения этого уравнения, удовлетворяюшие двум дополнительным соотношениям вида

$$
\begin{aligned}
{[R \otimes 1 T(x) 1 \otimes R, P] } & =0, \\
T(x) R \otimes 1 P T(1-x) & =\zeta R \otimes R,
\end{aligned}
$$

где $P$ есть оператор перестановки в $U \otimes U$, а оператор $R \in \operatorname{End}(U)$ таков, что

$$
R^{3}=1
$$

Из решения системы уравнений (8)-(11) можно построить решение системы уравнений (1)-(4) следуюшим образом. Определим пространство $V \equiv\left\{f: \mathbb{R}_{+}^{2} \rightarrow U\right\}$, где $\mathbb{R}_{+}=\{x \in \mathbb{R} \mid x>0\}$, и отождествим $V^{\otimes n}$ с $\left\{f: \mathbb{R}_{+}^{2 n} \rightarrow U^{\otimes n}\right\}$. Тогда операторы $\mathbf{T} \in \operatorname{End}(V \otimes V)$ и $\mathbf{R} \in \operatorname{End}(V)$, определяемые соотношением

$$
\mathbf{T} f(\mathbf{x}, \mathbf{y})=T\left(\frac{x_{1} y_{2}}{x_{1} y_{2}+x_{2}}\right) f(\mathbf{x} \cdot \mathbf{y}, \mathbf{x} * \mathbf{y}), \quad \mathbf{R} f(\mathbf{x})=R f(\hat{\mathbf{x}})
$$

где для любых $\mathbf{x}=\left(x_{1}, x_{2}\right), \mathbf{y}=\left(y_{1}, y_{2}\right), x_{i}, y_{i} \in \mathbb{R}_{+}$, функция $f(\mathbf{x}, \mathbf{y}) \in U \otimes U$ и по определению

$$
\begin{aligned}
\mathbf{x} \cdot \mathbf{y} & :=\left(x_{1} y_{1}, x_{1} y_{2}+x_{2}\right), \quad \mathbf{x} * \mathbf{y}:=\left(\frac{y_{1} x_{2}}{x_{1} y_{2}+x_{2}}, \frac{y_{2}}{x_{1} y_{2}+x_{2}}\right), \\
\hat{\mathbf{x}} & :=\left(\frac{x_{2}}{x_{1}}, \frac{1}{x_{1}}\right), \quad f(\mathbf{x}) \in U,
\end{aligned}
$$

удовлетворяют соотношениям (1)-(4). 
Пример 2. Пусть $U=L^{2}(\mathbb{R})$, а операторы $\mathbf{p}$ и $\mathbf{q}$ реализуют алгебру Гейзенберга самосопряженных операторов

$$
[\mathbf{p}, \mathbf{q}]=\frac{1}{2 \pi i}
$$

При $0<x<1$ и $y \in \mathbb{R}$ определим функцию

$$
\phi_{x}(y)=\exp \left(-i \pi x y^{2}\right)
$$

Она удовлетворяет тождеству пятиугольника

$$
\phi_{x}(\mathbf{q}) \phi_{y}(\mathbf{p})=\phi_{(y-x y) /(1-x y)}(\mathbf{p}) \phi_{x y}(\mathbf{p}+\mathbf{q}) \phi_{(x-x y) /(1-x y)}(\mathbf{q}) \text {. }
$$

Тогда операторы

$$
T(x)=\exp (2 \pi i \mathbf{p} \otimes \mathbf{q}) \phi_{x}(\mathbf{q} \otimes 1+1 \otimes(\mathbf{p}-\mathbf{q}))
$$

и

$$
R=\exp \left(2 \pi i \mathbf{q}^{2}-\frac{\pi i}{3}\right) \exp \left(\pi i \mathbf{p}^{2}\right) \exp \left(\pi i \mathbf{q}^{2}\right)
$$

удовлетворяют системе уравнений (8)-(11) при

$$
\zeta=\exp \left(-\frac{\pi i}{6}\right)
$$

Из уравнения (5) следует, что соответствующий центральный заряд есть четное целое число. Если исключить аналоги нефизических степеней свободы квантовой теории Тейхмюллера, то центральный заряд оказывается нечетным целым числом, что согласуется с интерпретацией в терминах свободной бозонной конформной теории поля. В действительности этот пример связан с бесконечномерным решением ограниченного соотношения звезда-треугольник Бажанова и Бакстера [4], которое возникает в трехмерной свободной бозонной модели на кубической решетке.

ПримеР 3. Пусть $U=\mathbb{C}^{N}$ при некотором целом $N \geqslant 2$, и пусть целое число $M$ является взаимно простым с $N$ и таким, что $|M| \leqslant N-1$ и $M+N=1(\bmod 2)$. Определим

$$
\theta:=\exp \left(i \pi \frac{M}{N}\right)
$$

Для любой матрицы $E$ такой, что $E^{N}=-1$, и любого $x$ из интервала $0<x<1$ компактный (или циклический) квантовый дилогарифм определяется следуюшим образом $[5,6]$ :

$$
\Psi_{x}(E):=\prod_{j=1}^{N-1}\left((1-x)^{1 / N}-x^{1 / N} E \theta^{-2 j}\right)^{j / N}
$$

где числа $(1-x)^{1 / N}$ и $x^{1 / N}$ действительны и положительны, а ветвь корня $N$-й степени выбирается из условия $\Psi_{0}(E)=1$. Эта функция удовлетворяет функциональному уравнению

$$
\Psi_{x}\left(\theta^{-2} E\right)=\left((1-x)^{1 / N}-x^{1 / N} E\right) \Psi_{x}(E)
$$


тождеству пятиугольника

$$
\Psi_{x}(F) \Psi_{y}(E)=\Psi_{(y-x y) /(1-x y)}(E) \Psi_{x y}(-E F) \Psi_{(x-x y) /(1-x y)}(F),
$$

где

$$
E F=\theta^{2} F E, \quad E^{N}=F^{N}=-1,
$$

и соотношению инверсии

$$
\Psi_{x}\left(-\theta^{2 k-1}\right) \Psi_{1-x}\left(-\theta^{-2 k-1}\right)=\theta^{-k^{2}} \exp \left(-i \pi A_{M, N}\right),
$$

где

$$
A_{M, N}=-A_{-M, N}=N^{-1} \sum_{k=1}^{N-1} k\left(\left\{\alpha_{k}\right\}_{1}-\left\{\left[\alpha_{k}\right]_{1}\right\}_{2}\right), \quad \alpha_{k} \equiv(2 k+1) \frac{M}{N} .
$$

Здесь $[x]_{y} \in \mathbb{Z}$ и $0 \leqslant\{x\}_{y}<y$ - соответственно целая и дробная части числа $x \in \mathbb{R}$ относительно числа $\mathbb{R} \ni y>0: x=\{x\}_{y}+y[x]_{y}$. В частности,

$$
A_{1-N, N}=\frac{1}{6}\left(N-N^{-1}\right), \quad A_{1, N}=-\frac{1}{12}\left(N+2 N^{-1}\right) .
$$

Введем матрицы $\theta^{2 a}, \theta^{2 b} \in \operatorname{End}(U)$, удовлетворяющие соотношениям

$$
\theta^{2} \theta^{2 a} \theta^{2 b}=\theta^{2 b} \theta^{2 a}, \quad \theta^{2 a N}=\theta^{2 b N}=1 .
$$

Тогда отображения

$$
T(x)=\Psi_{x}\left(-\theta^{-2 b} \theta^{2 a} \otimes \theta^{2 b}\right) \theta^{2 b \otimes a}
$$

и

$$
R=\exp \left(i \frac{2 \pi}{3}\left(2 A_{M, N}+B_{M, N}\right)\right) \theta^{2 a^{2}} \theta^{b^{2}} \theta^{a^{2}}
$$

где

$$
B_{M, N}=-B_{-M, N}=N^{-1} \sum_{k=1}^{N-1} k\left(\left\{\beta_{k}\right\}_{1}+\left\{\left[\beta_{k}\right]_{1}\right\}_{2}-1\right), \quad \beta_{k} \equiv 2 k \frac{M}{N}
$$

удовлетворяют уравнениям (8)-(11) при

$$
\zeta=\exp \left(i \frac{\pi}{3}\left(B_{M, N}-A_{M, N}\right)\right)
$$

Из уравнения (5) вытекает следующая формула для центрального заряда:

$$
c_{M, N}=24\left(A_{M, N}-B_{M, N}\right) \quad(\bmod 2) .
$$

В частности, принимая во внимание уравнение (12) и соотношение

$$
B_{1-N, N}=B_{1, N}=\frac{(N-1)(N-2)}{6 N},
$$

получаем

$$
c_{1-N, N}=c_{1, N}+6 N=12\left(1-N^{-1}\right)=-\frac{12}{N} \quad(\bmod 2) .
$$


Этот результат согласуется с удвоенным центральным зарядом $S U(2)_{N-2}$ конформной теории поля Весса-Зумино-Виттена- Новикова:

$$
2 c_{S U(2)_{N-2}}=\frac{6(N-2)}{N}=-\frac{12}{N} \quad(\bmod 2) .
$$

Данньй факт, очевидно, отражает связь межу циклическим квантовым дилогарифмом и неунитарным вариантом квантовой теории Тейхмюллера [1], которая, в свою очередь, предположительно связана с $S L(2, \mathbb{C})$-теорией Черна-Саймонса (через комплексификацию $S L(2, \mathbb{R}))^{1}$.

\section{PЕЗЮME}

Система операторных соотношений (1)-(4), приводящая к проективным представлениям групп классов отображений поверхностей с отмеченными точками, возможно, допускает много различных решений. Два примера решений из трех, представленных в данной работе, соответствуют двум случаям квантового дилогарифма - компактному и некомпактному. В случае компактного $q$-дилогариффма проективный множитель отвечает рациональному центральному заряду алгебры Вирасоро, задаваемому уравнением (13). Интересно отметить, что частное значение (14) согласуется с удвоенным центральным зарядом $S U(2)_{N-2}$-конформной теории поля Весса-Зумино-Виттена-Новикова.

\section{Список литературы}

[1] R. M. Kashaev. Lett. Math. Phys. 1998. V. 43. № 2. P. 105-115; q-alg/9705021.

[2] R. M. Kashaev. Liouville central charge in quantum Teichmüller theory. PDMI Preprint 24/1998. St-Petersburg: PDMI, 1998; hep-th/9811203.

[3] L. D. Faddeev. Lett. Math. Phys. 1995. V. 34. P. 249-254; hep-th/9504111.

[4] V. V. Bazhanov, R. J. Baxter. J. Stat. Phys. 1993. V. 71. P. 839-885.

[5] V.V. Bazhanov, N. Yu. Reshetikhin. Remarks on the quantum dilogarithm. Preprint ANU/ SMS/MRR-005-95. Canberra: Australia Nat. Univ., 1995; J. Phys. A. 1995. V. 28. № 8. P. 2217-2226.

[6] L. D. Faddeev, R. M. Kashaev. Mod. Phys. Lett. A. 1994. V. 9. P. 427-434; hep-th/9310070.

\footnotetext{
1) Точнее, следует исключить нефизические степени свободы, отвечающие связям в квантовой теории Тейхмюллера. Это легко сделать при нечетных $N$; в результате центральный заряд, рассматриваемый по модулю 2 , остается неизменным.
} 